Cómo citar este artículo: Bonomi López, V. Políticas penitenciarias y Derechos Humanos en el primer gobierno del Frente Amplio. Ley de Humanización de Cárceles, un ensayo antipunitivista en el Uruguay de 2005. Fronteras 14 (1): 14-27, enero-junio 2020.

\title{
Políticas penitenciarias y Derechos Humanos en el primer gobierno del Frente Amplio. Ley de Humanización de Cárceles, un ensayo antipunitivista en el Uruguay de 2005
}

\section{Penitentiary policies and Human Rights in the first government of the Frente Amplio. Prison Humanization Law, an anti- punitive trial in Uruguay in 2005}

Valeria Bonomi López ${ }^{1}$

\begin{abstract}
Resumen
El siguiente artículo es una aproximación al momento político que puso en agenda y llevó más tarde a la aprobación de la Ley de Libertad Provisional Anticipada en Uruguay, conocida como Ley de Humanización de Cárceles. Se utilizó como marco analítico el modelo de corrientes múltiples de John Kingdon (1995). Se trata de un trabajo descriptivo basado en análisis documental y entrevistas en profundidad sobre un momento en el que la crisis del sistema penitenciario pasó a ocupar un lugar central en la agenda pública, dando inicio a una serie de debates y reformas sobre el sistema que llegan hasta hoy.
\end{abstract}

Palabras clave: cárceles, derechos humanos, Uruguay, Frente Amplio, John Kingdon

\begin{abstract}
The following article approximates to political moment that places on the public agenda and later led to the approval of the Provisional Early Freedom in Uruguay, known as the Prison Humanization Law. John Kingdon's (1995) multiple streams framework was used for the analytical process. This is a descriptive work based on documentary analysis and in-depth interviews about a time when the crisis of the prison system took centre stage in the public agenda, beginning a series of debates and reforms on the system that reach today.
\end{abstract}

Keywords: Prisons, human rights, Uruguay, Frente Amplio, John Kingdon

1 Licenciada en Ciencia Política. Facultad de Ciencias Sociales. Universidad de la República. Correo electrónico: val. bonomi@gmail.com 


\section{Introducción}

Desde la salida de la última dictadura cívico-militar, en 1985, el Frente Amplio ${ }^{2}$ sostuvo una postura crítica ante las políticas de endurecimiento de las penas. Desde su rol de principal fuerza política de oposición, se concibe la seguridad ciudadana como un derecho humano; y dentro de esta misma temática propone una reforma sustancial del sistema penitenciario que tiene como principal objetivo su humanización. Asumir el gobierno, y por ende tomar las riendas de la seguridad, pronto genera tensiones en el discurso previamente sostenido.

Tras obtener su primera victoria presidencial en octubre de 2004, la llegada de esta fuerza política -cercana a la izquierda- al gobierno, si bien resulta novedosa, "no parece producir traumas mayores" (Caetano et al., 2008: 469). Pese a tener mayorías parlamentarias, el gobierno invita a sus adversarios a compartir posiciones en el gabinete, así como también a negociar las nuevas orientaciones de la política pública. Dado que esta negociación no prospera, finalmente el Poder Ejecutivo (PE) se conforma por integrantes de las propias filas del partido de gobierno. El Ministerio del Interior fue ocupado inicialmente por el Dr. José Díaz Chávez, quien asumió el compromiso con el presidente de desempeñar el cargo por dos años, considerado tiempo suficiente para las primeras reformas que proponía llevar adelante desde esta cartera.

En setiembre de 2005, se aprueba la ley N. ${ }^{\circ}$ 17.897 de Libertad Provisional Anticipada, conocida como Ley de Humanización y Modernización del Sistema Carcelario. Para el período 2010-2015 se modifican las funciones del Instituto Nacional de Rehabilitación (INR) a través de la ley N. ${ }^{\circ} 18.719$ de Presupuesto Nacional, y hoy en día se discute si para un siguiente gobierno del Frente Amplio sería viable trasladar el sistema penitenciario desde la esfera del Ministerio del Interior al Ministerio de Educación y

2 Coalición de partidos del espectro político centro izquierda uruguayo, fundada en el año 1971.
Cultura, así como la aprobación de una ley integral para el sistema penitenciario, entre otras reformas. Estas últimas propuestas, aunque tienen absoluta vigencia, quedan por fuera del marco temporal considerado en este proyecto.

Si bien hay un debate que permanece y resuena en la agenda política desde distintos lugares, el sistema carcelario presenta hasta ahora diversas problemáticas que implican la sistemática violación de los derechos humanos, tanto para personas privadas de libertad (de aquí en más, PPL), como para los funcionarios, que van desde la sobrepoblación y hacinamiento, y malos tratos, hasta recurrentes denuncias de torturas. Por una parte, las tasas de prisionización en Uruguay son de las más altas de la región, ${ }^{3}$ y, por otro lado, la tendencia desde ciertos sectores de la sociedad -y también de la clase política- es al reclamo del endurecimiento de penas y de las políticas de seguridad. Uruguay no está ajeno a lo que se define como populismo punitivo, al menos desde el retorno a la democracia, en un proceso lento que se vuelve evidente en el año 1995 con la aprobación de la ley N. ${ }^{\circ} 16.707$ de Seguridad Ciudadana y las posteriores modificaciones de urgencia a esta mediante la ley $\mathrm{N} .^{\circ} 17.243$ del año 2000, que claramente entra en tensión con cualquier intento de humanizar la reclusión.

Es así que, para abordar las primeras modificaciones al sistema penitenciario en Uruguay desde los gobiernos de izquierda, se toma en cuenta los ensayos de la izquierda en el primer gobierno del Frente Amplio, específicamente en la gestión de Díaz, y se plantea la siguiente pregunta: ¿Cómo fue el proceso que pone en agenda a las políticas penitenciarias en el año 2005?

Para ello utiliza el modelo de corrientes múltiples del politólogo John Kingdon (2003) como aproximación a las modificaciones al sistema car-

3 Uruguay es el sexto país en América Latina con la mayor tasa de presos (321 PPL cada 100.000 habitantes), luego de El Salvador, Cuba, Panamá, Costa Rica y Brasil, y ocupa el lugar 27 a nivel mundial, según informe del año 2017 del Institute for Criminal Policy Research (ICPR) de la Universidad de Londres. 
celario. A modo de aclaración, no hay en Uruguay aproximaciones desde la ciencia política al castigo como política pública; por lo tanto, el abordaje es, por un lado, una gran deuda, así como también es un enorme desafío.

\section{Marco analítico}

Las políticas públicas son el medio para la resolución de problemas que se identifican como públicos. En la medida en que no pueden ser resueltos colectivamente, el Estado interviene, al menos para corregirlos, es decir, es la solución a un problema público reconocido como tal en la agenda gubernamental. La inacción estatal también es una forma de intervenir; es optar por el mantenimiento del statu quo.

Las Corrientes Múltiples de Kingdon (2003) parten de la complejidad, desorden e imprevisibilidad de los procesos políticos (Sanjurjo, 2019) y proponen un modelo analítico del proceso de formulación de políticas que consta de tres familias de procesos que fluyen a través del sistema político:

1. corriente de problemas: que refiere a las situaciones que los ciudadanos quieren cambiar, demandas o reivindicaciones que adquieren estatus público;

2. corriente de las políticas públicas o soluciones: en tanto proceso de construcción y selección de alternativas que se confrontan o compiten en la arena pública con el propósito de resolver el problema. En esta corriente los actores (estatales y no estatales) compiten porque su solución ingrese a la agenda;

3. y finalmente la corriente política, que está conformada por tres componentes: el ánimo nacional, las campañas y la rotación de la administración. El primero y el último son los que tienen el efecto más fuerte.
Las tres corrientes son independientes y poseen sus propias reglas, pero confluyen en un punto crítico en el tiempo dando lugar a una "ventana de oportunidad", precisamente la oportunidad en que los emprendedores de políticas pueden proponer e impulsar sus soluciones al problema en cuestión. Las ventanas de oportunidad se abren tanto por problemas urgentes, así como por acontecimientos en la corriente política. Esta apertura suele ser de corta duración, y puede ser tan predecible, como el presupuesto anual, o impredecible, como una salida vía política pública a la ocurrencia de una catástrofe (Zahariadis, 2010).

El modelo Kingdon se centra en la etapa predecisional en la que interactúan diversidad de actores, que pueden ser tanto formales como informales, individuales o colectivos, y se pueden encontrar en cualquiera de los tres flujos de corrientes múltiples, afectando el resultado en la posterior definición, diseño y formulación de las políticas. Estos actores se clasifican en dos grupos: al interior del gobierno o fuera de este; y además distingue los recursos e incentivos que puedan movilizar para el establecimiento de la agenda o la formulación de alternativas. Los primeros tendrán mayor injerencia en la agenda; los segundos, en las alternativas. Y algunos casos, como los legisladores o funcionarios del $\mathrm{PE}$, tienen incidencia en ambas. Dependiendo del lugar en donde estén, los emprendedores de políticas acceden a espacios de toma de decisión, movilizan recursos y formulan estrategias para que su propuesta se transforme efectivamente en una política pública.

El modelo postula que las ventanas de oportunidad se abren por la convergencia de las corrientes previas, $y$, tal como se mencionó, no permanecen mucho tiempo abiertas. En este sentido las políticas son formuladas en condiciones de ambigüedad, y el factor temporal es fundamental: la decisión política lejos está de ser racional, lógica y secuencial, sino que es azarosa y coyuntural. 
Figura 1: Diagrama del modelo de corrientes múltiples.

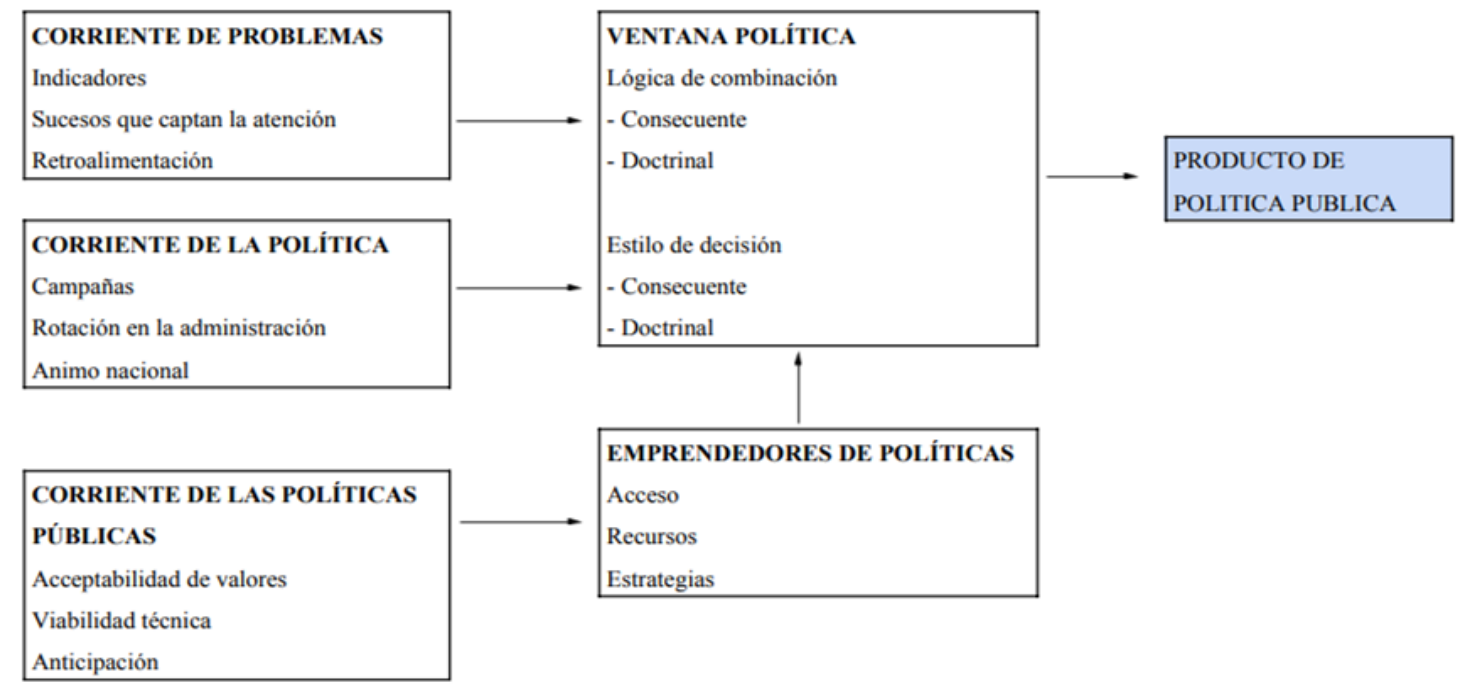

Fuente: Adaptado de Zahariadis (2010)

\section{Consideraciones metodológicas}

Para cumplir con el objetivo de reconstruir el momento político que llevó a la aprobación de la ley N. ${ }^{\circ} 17.897$ a través del modelo de corrientes múltiples se consideran fuentes documentales primarias: el Informe de la Comisión Honoraria de Mejoramiento del Sistema Penitenciario de 1996, el documento del IV Congreso Héctor Rodríguez del Frente Amplio del año 2003, la declaración de Emergencia Humanitaria para el Sistema Penitenciario de parte del Dr. Tabaré Vázquez en el acto de asunción presidencial en marzo de 2005, y finalmente el Informe del Poder Ejecutivo que acompañó al proyecto de ley de Libertad Provisional Anticipada y los informes de comisión parlamentaria que llevaron a la aprobación de dicha ley en setiembre de 2005.

Asimismo se realizaron entrevistas en profundidad semiestructuradas a actores relevantes del momento: Álvaro Garcé, excomisionado parlamentario para el sistema penitenciario; Rafael Paternain, exfuncionario del Observatorio de Violencia y Criminalidad del Ministerio del Interior; Juan Faroppa, exsubsecretario del Ministerio del Interior; Enrique Navas, exdirector de Cárceles, y finalmente a María Noel Rodrí- guez, asesora del Ministerio del Interior en los primeros años de gestión del Frente Amplio. Se intentó concretar una entrevista con Díaz, pero complicaciones de salud impidieron que se llevase a cabo.

\section{De cárceles y castigos}

En las sociedades contemporáneas se ha extendido el castigo desde Estado a la sociedad. Cada vez hay más agencias que hacen del castigo su forma de intervención. No solamente se castiga con cárcel, con policías, sino también en escuelas u hospitales. Los malos tratos son ejercicios del castigo. Estamos atravesando un "momento punitivo" que se traduce en un aumento del castigo en sus diversas formas o aristas: socioeconómicas, culturales, políticas e incluso morales. El aumento del número de PPL no necesariamente se corresponde con el aumento de la criminalidad y de la delincuencia. Por un lado, hay cierta intolerancia por parte de la sociedad y más sensibilidad a los desórdenes y a las desviaciones. Por el otro, hay una manipulación de los políticos de esa inquietud de la población alrededor del crimen con propuestas de más castigos, más sentencias 
de prisión y la construcción de más cárceles-, que es lo que se llama "populismo penal". La combinación de la intolerancia y el populismo penal habilita la posibilidad de una desconexión entre el aumento de PPL y la realidad de la criminalidad y lo que caracteriza al punitivismo o populismo punitivo (Fassin, 2018).

En simultáneo, y como parte de este momento punitivo, se ha naturalizado la creencia de que la única forma de "hacer justicia" y "pagar" por un delito cometido es la reclusión penitenciaria. La privación de libertad en tanto solución eficaz al problema de inseguridad o el delito se asocia, al menos, con cuatro razones. En primer lugar, la persona encerrada queda inhabilitada a tener una conducta delictiva. Segundo: la privación de libertad es suficientemente costosa para reiterar esa conducta. Tercero: la prisión supone un espacio de educación y persuasión para no querer delinquir nuevamente. Y, por último, la persuasión también aplica al resto de la sociedad aunque la investigación criminológica de los últimos años indica lo contrario: la cárcel no solo parecería ser ineficiente en el cumplimiento de estos cuatro motivos, sino que además resulta criminógena (Bardazano et al., 2015).

De acuerdo con Garland (2012) el declive del ideal de rehabilitación ha sido uno de los cambios más importantes en la política penal de los últimos años: una reducción del énfasis puesto en la rehabilitación como objetivo de la privación de libertad y modificaciones en legislación regulatoria de condenas que se alejan de los programas de tratamiento durante las condenas. Si bien señala que en ocasiones los programas re se aplican, no forman parte de las prácticas dominantes ni tampoco se señalan entre los principales objetivos de las medidas penales. Las prácticas rehabilitadoras finalmente se subordinan a otros objetivos: la retribución, la incapacitación y la gestión de riesgo.

La cárcel y su gestión ponen de manifiesto el modelo humano en juego, el tipo de sujeto deseable y castigable. En este sentido, el castigo es "una institución social que se revela, en efecto, como un notable parámetro de análisis de las sociedades, de los afectos que las atraviesan y los valores de las que son portadoras" (Fassin, 2018: 44) y deviene por lo tanto en un subsistema particularmente artificial ya que con su intervención corta con la historia de vida de la persona privada de libertad. El encierro es por ello ilusorio en tanto las posibilidades de modificar comportamientos y de mejoramiento de las personas. La cárcel interpela al delincuente que es encerrado, pero también la identidad del ciudadano respetuoso de la ley es parte de este mismo marco simbólico amenazante (Calderón, 2015).

La cárcel aísla, pero no separa, es parte de la sociedad en la que se inserta. Las políticas penales son una expresión de cierta gama de sentimientos colectivos. Desde hace años el temor al delito viene adquiriendo mayor relevancia. Lo que inicialmente se veía como una cuestión situacional localizada se ha ido trasladando desde lo discursivo, y transformando en un problema social fundamental. Junto a este temor, se instala la desconfianza en el sistema político para resolver los asuntos de seguridad, por lo que también se transforma el discurso político (y consecuentemente las políticas) apelando a ese público temeroso, desconfiado y molesto. La política criminal resulta atravesada por el enojo colectivo que reclama más la retribución que la búsqueda de soluciones estructurales y más justas (Garland, 2012).

En este sentido, las cárceles devienen en un espacio de concentración de la exclusión social. Ya Foucault (2008) señalaba que el diseño del castigo venía de la mano del desarrollo de la sociedad capitalista. La condena pública de un delito y la decisión posterior de castigo no solamente atienden a sus características, sino que dependen también de las relaciones de poder en las cuáles se insertan. En esta línea, Fassin (2018) sostiene que el acto de castigar debería de ser equitativo: "la justicia justa", como si no hubiera marcadas disparidades etnorraciales y socioeconómicas dentro del sistema penitenciario. La relación entre crimen y castigo es menos unívoca de lo deseable y la demografía carcelaria es prueba de ello. La represión selectiva de ciertas categorías de ilegalismos y también de ciertas poblaciones 
juega un rol importante en la reproducción de las disparidades sociales.

\section{Los problemas: Uruguay hacia las reformas de 2006}

En lo que respecta a Uruguay, el desarrollo de políticas públicas penitenciarias se ha encontrado con esfuerzos aislados e inconexos, producto de emergencias coyunturales que requerían intervención urgente de los tomadores de decisiones. "En las últimas tres décadas no se han desarrollado políticas integrales de largo plazo para enfrentar el problema penitenciario de manera coordinada y sometida a procesos de evaluación" (González et al., 2015: 128).

Previo a 2005, en el periodo 1995-2004, se identifican cambios en el sistema criminal del Uruguay bastante significativos: la aprobación de las leyes 16.707 y 17.243 de corte punitivo crean nuevas figuras delictivas e incrementan la severidad de las penas para delitos ya vigentes, lo cual favoreció la inflación penitenciaria y el colapso posterior del sistema (González et al., 2015).

Gráfico 1. Personas privadas de libertad cada 100.000 habitantes (eje izq.) y porcentaje de hacinamiento (eje der.), 1988-2014

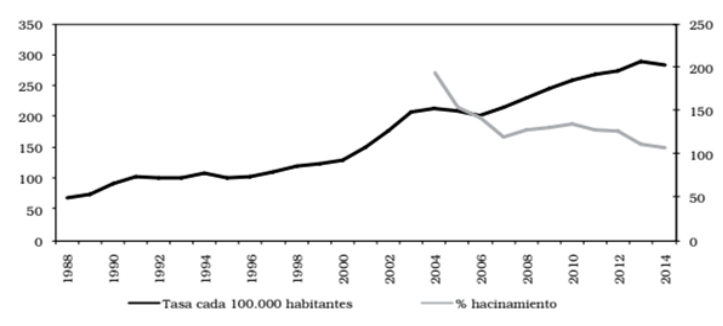

Nota: no se disponen datos de hacinamiento antes de 2004.

Fuente: González, Rojido y Trajtenberg (2015), elaborado con datos del Observatorio de Violencia y Criminalidad del Ministerio del Interior

En este mismo período también se crea la Comisión Honoraria para el Mejoramiento del Sistema Penitenciario, se realiza el Primer Censo Nacional de Población Reclusa en 1999, se crea el Centro Nacional de Rehabilitación en 2002 y también el Comisionado Parlamentario para el
Sistema Penitenciario, cuya función es de monitoreo y asesoramiento a los legisladores acerca de las condiciones del sistema penitenciario y sobre el cumplimiento de la normativa referente a la PPL, tanto la nacional como la internacional.

Las políticas de prevención del delito y la creación de nuevos espacios institucionales dentro del Ministerio del Interior daban señales de un cierto fortalecimiento estatal, estrategias de vinculación con la ciudadanía además de nuevos abordajes a temas de violencia doméstica; sin embargo, no hubo una estrategia política sistemática, organizada y de largo alcance. La llegada de la izquierda al gobierno resignifica estos procesos, al menos colocándolos en un lugar de relevancia además de renovando sus enfoques.

La política de seguridad fue un terreno bastante exploratorio para el Frente Amplio una vez asumido el gobierno en 2005; asimismo la historia reciente estaba marcada por una fuerte polarización entre las fuerzas de seguridad del Estado y muchos actores que ahora llegaban al gobierno. A su vez, la designación del ministro fue crucial para el Dr. Vázquez, quien enfocó su búsqueda en una persona de "fuerte temple político, capacidad de negociación y habilidades para negociar una transición compleja e incierta" (Vila, 258: 2016).

La ley N. ${ }^{\circ} 16.707$ del año 1995 crea en su artículo 34 la Comisión Honoraria de Mejoramiento del Sistema Carcelario con el fin de asesorar al Poder Ejecutivo en esta materia. Comienza sus tareas el 28 de setiembre de 1995, que culminan con la presentación de su informe en junio de 1996. Este es extenso, y un análisis detallado excede a los propósitos de este trabajo, pero se consideran sus lineamientos generales, dado que no fueron tenidos en cuenta hasta que el Frente Amplio los incluye en su programa de gobierno y luego en la discusión de la ley de Libertad Provisional Anticipada. El informe completo se estructura en áreas temáticas acorde al cumplimiento de las Reglas Mínimas para el tratamiento de los reclusos de las Naciones Unidas, hoy en día reformuladas como Reglas Nelson Man- 
dela. ${ }^{4}$ De ello surge un diagnóstico crítico de la situación de las cárceles y por ende también de su población:

1. inadecuación de los edificios;

2. superpoblación y hacinamiento;

3. ausencia de capacitación de funcionarios. Utilización de la función penitenciaria como sanción o desvalorización del funcionario;

4. distribución inadecuada de los funcionarios y desorganización de los equipos técnicos;

5. corrupción, tráfico y consumo problemático de drogas y alcohol;

6. violencia física institucional legitimada, y

7. desvalorización de los derechos de la población: carencias alimentarias, de servicios de salud, visitas, trabajo, educación y recreación.

En el año 2003, el IV Congreso Héctor Rodríguez, del Frente Amplio, propone las bases programáticas para lo que sería el próximo gobierno. En el documento que surge del Congreso se determina como prioritaria la situación del sistema penitenciario. Al respecto el diagnóstico que se realiza es breve y sumamente crítico de los gobiernos anteriores. Señala a las cárceles como un ámbito de violación permanente de los derechos fundamentales de los internos en contradicción con el artículo 26 de la Constitución ${ }^{5}$ y la concepción humanitaria de la convivencia democrática. El documento denuncia el creciente deterioro del sistema tanto en el aspecto alimenticio como sanitario desde la salida de la última dictadura cívico-militar, y califica de inhumano el trato hacia la PPL.

4 Se trata de un conjunto de 122 reglas aprobadas por Asamblea General de las Naciones Unidas, referidas a la protección de las garantías básicas de las personas privadas de libertad, que ponen especial énfasis en el respeto a la dignidad humana, la prohibición de la tortura. No vuelven a ser mencionadas en los demás documentos analizados.

5 A nadie se le aplicará la pena de muerte. En ningún caso se permitirá que las cárceles sirvan para mortificar, y sí solo para asegurar a los procesados y penados, persiguiendo su reeducación, la aptitud para el trabajo y la profilaxis del delito. Artículo 26 de la Constitución de la República.
Un aspecto fundamental de este proceso es el acto de asunción del gobierno frenteamplista, el $1^{\circ}$ de marzo de 2005, en el que Tabaré Vázquez decretó la emergencia humanitaria del sistema penitenciario a la vez que daba otras señales de un marcado enfoque de derechos humanos en lo relativo a las políticas de seguridad ciudadana.

"La grave situación existente en las cárceles y la falta de atención a las víctimas del delito nos determina declarar, a partir del día de hoy, el estado de emergencia humanitaria en todo el país (...) Tener en cuenta, uruguayas y uruguayos, que la tasa media mundial de población carcelaria de un país respecto a la población total del mismo es de 0,5 por mil. De acuerdo a esa estadística, Uruguay debería tener unos 1500 presos; hoy tiene casi 7000, la mayoría jóvenes y pobres, y la pobreza no se combate construyendo cárceles para jóvenes".

Pronunciadas estas palabras por Tabaré Vázquez ante el pleno del Poder Legislativo, se lanza un claro mensaje sobre la necesidad de atacar urgentemente la situación crítica del sistema penitenciario. El nuevo gobierno da cuenta del estado de las prisiones y de sus habitantes, y se propone su mejoría entre sus prioridades.

En julio del 2005 el Poder Ejecutivo remite al Parlamento el proyecto de ley de régimen excepcional para humanización del sistema penitenciario. Inmediatamente es derivado a la Comisión de Constitución, Códigos, Legislación General y Administración, integrada con la Comisión de Derechos Humanos. Para este proyecto el Poder Ejecutivo busca asesoramiento y apoyo de diversas instituciones: la Suprema Corte de Justicia, el Instituto de Derecho Penal de la Facultad de Derecho de la Universidad de la República, la Asociación de Magistrados y de Magistrados Fiscales, Defensores de Oficio, además de algunas unidades del Ministerio del Interior vinculadas a lo penitenciario. En tal sentido moviliza recursos y busca consensos dentro y fuera del gobierno para obtener apoyos en la promoción de esta política.

Dicho proyecto, que se presenta como parte de un conjunto de medidas para superar a la que 
define como "la peor crisis humanitaria" dentro de sistema penitenciario en nuestra historia reciente, expone como introducción un diagnóstico severo respecto de las cárceles en Uruguay, donde se exponen diversas problemáticas que además coinciden con las relevadas por la Comisión durante 1996, a saber: precaria situación de los establecimientos de detención, donde el hacinamiento supera los estándares internacionales, la orfandad alimenticia pone en riesgo la nutrición de la población reclusa, y los servicios sanitarios padecen los efectos de la insuficiente asignación presupuestal.

Cuadro 1. Personas presas. Tasas cada 100.000 habitantes. 1994-2004 (no hay datos disponibles para 1995)

\begin{tabular}{|c|c|c|c|c|c|c|c|c|c|}
\hline 1994 & 1996 & 1997 & 1998 & 1999 & 2000 & 2001 & 2002 & 2003 & 2004 \\
\hline 100 & 101 & 106 & 119 & 121 & 128 & 146 & 166 & 202 & 212 \\
\hline
\end{tabular}

Fuente: Proyecto de ley de Libertad Provisional Anticipada

Este visible crecimiento de la población penitenciaria llevó a un hacinamiento crítico, con una densidad promedio a nivel nacional de 196, por lo que, conforme a los parámetros que tomaba el informe del Consejo de Europa, se trataba de un hacinamiento crítico. ${ }^{6}$ Esto al mismo tiempo conlleva a otras problemáticas, como la escasez alimentaria o la insatisfacción de las demandas sanitarias, patologías severas como VIH o bien pacientes psiquiátricos sin cobertura asistencial adecuada.

6 A nivel internacional el hacinamiento se mide mediante la densidad carcelaria cada 100 plazas (número de personas privadas de libertad sobre capacidad instalada, por 100). Según el parámetro utilizado por el Consejo de Europa, el hacinamiento se califica de "crítico" cuando la densidad es igual o superior a 120. Uruguay, en el año 2005, presentaba una densidad promedio nacional de 196, por lo que, de acuerdo con los parámetros internacionales, el hacinamiento se considera severamente crítico.

\section{Las soluciones: un proyecto controversial sin propuestas alternativas}

En esta segunda parte se presentan las soluciones planteadas al problema penitenciario extraídas de los mismos documentos analizados en el capítulo anterior.

Tal como se mencionó en el apartado anterior, la Comisión de Mejoramiento del Sistema Carcelario, en su informe al Poder Ejecutivo en 1996, realiza un severo diagnóstico y varias propuestas para iniciar un proceso a largo plazo de mejoramiento del sistema, cuyo objetivo rector es una cárcel mínima en cuanto a número de internos, donde prime el trato personal y humanitario, y sea posible el cumplimiento de los objetivos de rehabilitación y reeducación como ejes de la progresividad del sistema y la organización de la cotidianidad en el encierro. Los servicios de vigilancia, mantenimiento, administración, salud, educación y trabajo serían, asimismo, pautados para mejorar la convivencia y para que logren reducir los índices de violencia.

La primera propuesta refiere a la ubicación institucional: las cárceles deben salir de la órbita del Ministerio del Interior. Una segunda propuesta es el fortalecimiento institucional del Patronato Nacional de Encarcelados y Liberados aumentando su personal, mejorando los salarios, generando incentivos para el trabajo con los egresados del sistema, profesionalizando la tarea, entendiendo que la eficacia en la tutela es fundamental para prevenir la delincuencia y controlar la reincidencia.

La tercera parte del informe de la comisión se centra en la libertad provisional; si bien pone el acento en la larga duración de los procesos judiciales y no en el problema penitenciario per se, este es el antecedente más directamente relacionado a la ley de Libertad Provisional Anticipada, finalmente aprobada en 2005. Aquí se presenta un anteproyecto de ley a la Suprema Corte de Justicia solicitando liberación anticipada para aquellos casos en que:

1. la PPL hubiera sufrido prisión preventiva equivalente a la mitad de su pena requerida 
en la demanda acusatoria o impuesta en la sentencia;

2. si hubieran sido requeridas o impuestas medidas de seguridad eliminativas y han transcurrido en prisión preventiva las dos terceras partes de la pena; $y$

3. para aquellos casos en que el proceso se había iniciado hace tres años o más, y aún no existía acusación fiscal.

En el programa para el nuevo gobierno del Frente Amplio (2003) se dedica un espacio a la seguridad ciudadana y el rol de la Policía nacional, el Ministerio del Interior y también el sistema penitenciario. No es menor que el capítulo comienza definiendo la seguridad ciudadana como un derecho humano:

Los objetivos estratégicos del Encuentro Progresista-Frente Amplio en materia de seguridad ciudadana tienen como base el concepto de seguridad como derecho humano. Como tal, el Estado tiene el deber de garantizar su goce efectivo a todas las personas que habitan en su territorio sin ningún tipo de distinción.

En este sentido, el documento propone modificaciones y reestructuras dentro del Ministerio del Interior y de la Policía nacional, y además dedica un espacio a la necesidad de una reforma sustancial y prioritaria del sistema penitenciario, como un aspecto más para garantizar los derechos humanos tanto de habitantes libres como PPL.

El mencionado plan se centra en los siguientes objetivos a cumplir en el corto plazo:

1. asignar recursos para la humanización de las condiciones de reclusión, así como también de las condiciones laborales del personal penitenciario;

2. establecer un programa de capacitación a funcionarios, y

3. finalmente, centralizar todas las cárceles que dependen de las Jefaturas de Policía y que queden bajo la Dirección Nacional de Cárceles, Penitenciarías y Centros de Recuperación (esto se hará mucho más adelante).
Los cometidos de largo plazo deben seguir los lineamientos del Informe de la Comisión Honoraria de Mejoramiento del Sistema Carcelario. De este informe en el programa se destaca la creación de un Instituto Nacional de Cárceles, como servicio descentralizado, que pasaría a regir sobre todos los institutos de reclusión luego de un proceso gradual de traspaso; la formación de personal técnico, luego de la creación de un centro de formación y estudios, dependiente del Instituto, pero con convenios con la Udelar, ${ }^{7}$ y finalmente proponen un tratamiento personalizado de la PPL cuyos principios rectores serían su personalidad, dignidad y humanidad, incentivando el relacionamiento con la familia así como también preparando las condiciones de egreso del sistema.

El Poder Ejecutivo presenta el proyecto de ley de libertad provisional anticipada como una forma de atender la precaria situación del sistema penitenciario desde las libertades, la creación de una institución de protección a los derechos de las víctimas y la derogación de algunas disposiciones penales de la Ley de Seguridad Ciudadana y sus posteriores reformas. En este sentido se proponía modernizar el sistema penitenciario para finalizar con la creación del Instituto Nacional de Rehabilitación (INR).

En este sentido el proyecto se presenta como el paso inicial de una serie de profundas reformas al sistema penitenciario, a implementarse en tres fases: la primera consiste en superar el estado de emergencia brindando a la PPL condiciones dignas; la segunda etapa sería la unificación del sistema carcelario bajo la dirección de la Dirección Nacional de Cárceles, y, finalmente, la creación del INR fuera de la órbita del Ministerio del Interior.

Con respecto a la superación de la situación de emergencia, se señalan una serie de medidas que acompañan el proyecto de ley que van desde el relevamiento de información hasta la asignación de presupuesto para alimentos; en buena medida

7 El Centro de Formación Penitenciaria (Cefopen) se crea recién en 2010 junto con el Instituto Nacional de Rehabilitación (INR) y depende del Ministerio del Interior 
estos puntos son una reelaboración sumaria de las propuestas de la Comisión de 1996; aunque no todos finalmente se concretan; entendiendo que el proyecto es un primer paso para abordar la crisis penitenciaria, mas no es definitivo: las soluciones definitivas vendrían de la mano del fortalecimiento de las políticas sociales en concordancia con el discurso de marzo del presidente Tabaré Vázquez que sostiene que, si bien es necesario atender las emergencias del sistema, también es de orden ir a las causas más estructurales del problema, comprometiendo su gestión en el combate a la delincuencia y a sus causas.

La respuesta de las comisiones legislativas fue, por un lado, un informe de apoyo al proyecto de ley, tal como ya se mencionó, y dos informes en minoría que rechazan la votación del proyecto. Si bien los miembros informantes admitían que el sistema penitenciario se encontraba en situación de emergencia, no ven en la propuesta de ley -especialmente en los artículos vinculados a la libertad anticipada- una solución al problema de hacinamiento específicamente, ni tampoco a las condiciones de reclusión en general, además de que significa también un riesgo para los demás ciudadanos al incrementar inseguridad. Es en esta línea que ambos informes exponen los motivos por los cuales rechazan el proyecto, instando al pleno a no votarlo, sin generar una propuesta alternativa que, de hecho, no hubo.

\section{Las políticas: ley $\mathrm{N} .^{\circ} 17.897$}

La ley que se revisa a continuación es tanto resultado de la convergencia de todos los factores que se mencionaron, así como también una inclusión parcial de un marco humanitario relativo al sistema penitenciario en nuestro sistema legal. Esto significa que la ley no logra solucionar todos los problemas del sistema ni tampoco considera todas las soluciones que aparecen en distintos momentos del debate.

De la discusión parlamentaria, que tuvo una instancia en cada cámara, se extrae que los apoyos y rechazos al proyecto dan cuenta de la adhesión partidaria de los representantes: vale decir que el Frente Amplio contaba en su momento con ma- yorías parlamentarias disciplinadas y que además es característico de Uruguay que los partidos políticos permean cualquier tipo de debate, y esta no fue la excepción.

Desde el oficialismo se puso el énfasis en la protección de derechos de todos los ciudadanos y la responsabilidad del Estado en materia de reclusión, así como también se reitera en los discursos el fracaso de la lógica punitiva y su consecuente colapso del sistema penitenciario; se reitera también la pretensión de encaminarse hacia un nuevo paradigma de políticas de seguridad, y esta norma conformaría el mojón inicial en esa dirección. Desde filas de la oposición, se argumenta en contra del proyecto por considerarse insuficiente en cuanto a solución del problema penitenciario, además de exponer a la población a mayor riesgo de inseguridad con la liberación de PPL. Asimismo, hacen hincapié en las víctimas, y tornan la discusión en una cuestión dicotómica: delincuentes versus víctimas, o población civil, aunque esa dualidad estaba lejos del espíritu de la ley.

Si bien, finalmente, la discusión parlamentaria devino también simplificada, estuvo presente una mirada de derechos humanos (en la sesión en la Cámara de Representantes se menciona unas setenta veces); de fondo estaba la cuestión de ¿derechos para quién? Esto también permite entrever un debate más de fondo, de concepciones de seguridad pública, de los motivos, usos y fines del castigo, y por último también de un modelo de sociedad. Asimismo, se hace patente que no había consenso al respecto de las visiones del ministerio sobre cómo humanizar la privación de libertad y de si esta ley era la vía para hacerlo, revelando también lo antes mencionado al respecto de las políticas como resultado de procesos ambiguos.

En concordancia con el proyecto original del Poder Ejecutivo, la ley N. ${ }^{\circ} 17.987$, finalmente aprobada, puede dividirse en varias partes:

La primera -artículo 1 al $7^{\circ}$ - establece un régimen excepcional de libertad provisional anticipada que se aplicaría, por única vez, a los procesados y penados que estaban privados de libertad 
al $1 .^{\circ}$ de marzo de 2005, dejando por fuera a aquellos penados por cometer delitos graves (homicidios, violaciones, rapiña con arma de fuego, etcétera). Como condición, para el estudio del caso, se pedía el cumplimiento de al menos dos tercios de la pena impuesta si era mayor a 3 ańos; o bien al menos media pena si era de hasta 3 años de penitenciaría. El trámite se hacía vía judicial, con un plazo de hasta 60 días, y una vez concedida la libertad, la persona liberada quedaba bajo tutela del Patronato. El incumplimiento al régimen revocaba el beneficio.

En segundo lugar-artículos 8 y 9- se regula sobre medidas de seguridad provisionales al modificar el artículo 131 del Código Penal, permitiendo régimen de prisión domiciliaria para aquellas personas que fueran diagnosticadas con enfermedades graves, y mujeres con embarazo avanzado, así como también privados de libertad mayores de 70 años, con excepción de aquellos que hubieran cometido homicidios -con determinadas agravantes previstas en los artículos 311 y 312 del Código Penal-, violación o bien delitos previstos en el Estatuto de Roma de la Corte Penal Internacional, al que Uruguay adhiere en el año 2002 por la ley N. ${ }^{\circ} 17.510$, en referencia a los delitos de lesa humanidad.

En el tercer capítulo -artículo 10 y siguientesla ley modifica algunas disposiciones del Código Penal respecto de las salidas transitorias, la libertad condicional y la libertad anticipada, flexibilizándolas.

Otra parte de suma importancia en la nueva legislación -artículos 13 y 14- establece un régimen de redención de pena por días de trabajo o estudio, en una relación de un día cada dos. Para ello, las autoridades carcelarias determinarían los trabajos posibles a desarrollar en cada centro. Y, además, se preveía una cuota del 5\% de PPL en licitaciones públicas (para trabajos de peones o similares) y bonificaciones a empresas por recurrir a la bolsa de trabajo del PNEL.

Finalmente, creaba un Centro de Atención de víctimas del delito que se trataría como parte del presupuesto nacional en el marco de la estructura actual de la Dirección Nacional de Prevención
Social del Delito. Creaba también una comisión para la reforma del Proceso Penal y otra del Código Penal; ambas integradas por un representante del Poder Ejecutivo como presidente, de la Suprema Corte de Justicia, la Fiscalía de Corte, la Universidad de la República, la Asociación de Magistrados Judiciales, la Asociación de Magistrados Fiscales, la Asociación de Defensores de Oficio, el Colegio de Abogados del Uruguay, la Asociación de Funcionarios Judiciales, la Asociación de Actuarios Judiciales y el Ministerio de Economía y Finanzas.

\section{Los actores en el modelo y en el debate del proyecto de ley}

El emprendedor indiscutido de esta política pública es el Dr. José Díaz, como se mencionó antes, elegido por el Dr. Tabaré Vázquez para la Secretaría de Estado a cargo de la seguridad pública, por su capacidad negociadora y sus capacidades para iniciar una transición compleja. De las entrevistas se desprende que el equipo de gobierno entrante estuvo trabajando durante meses en el hotel presidente, preparándose para la gestión.

El Dr. Díaz asumió con tres compromisos bien específicos, y con el acuerdo de permanecer dos años en la gestión, dejar las primeras reformas delineadas y dar paso a alguien más que pudiera continuar: ${ }^{8}$ gestionar la transición, redefinir la función policial acotada a la seguridad interna y cumplir con los compromisos asumidos en tratados internacionales en materia de derechos humanos. Era fundamental para el gobierno entrante y especialmente para el ministro revertir la tendencia punitiva de los periodos anteriores (Vila, 2016).

Respecto al sistema penitenciario, los fines orientados por el ministro para cumplir durante el período eran: en primer lugar, cubrir las necesidades básicas de alimentación, salud, alojamiento y seguridad, tanto de la PPL como del personal

8 La secuencia de hechos y tensiones políticas a partir de la implementación de la ley 17.897 impidió que el ministro continuara a la cabeza del ministerio hasta el final del período, de todas maneras. 
penitenciario asignado a la custodia de los centros del sistema, así como también incentivar la participación de la PPL en actividades educativas, laborales y recreativas, ambos aspectos claramente reflejados en el contenido de la ley.

El nuevo ministerio desarrolló un sistema de comunicación transparente, tratando de contextualizar los problemas de seguridad con los fenómenos emergentes a nivel regional, presentando constantemente información a la ciudadanía desde el Observatorio de Violencia y Criminalidad del Ministerio, creado en agosto del año 2005, una forma de transparentar la gestión, además de una marca particular del Dr. Díaz.

\section{Consideraciones finales}

Lo anterior fue una aproximación al momento político que puso en agenda y llegó más tarde a la aprobación de la Ley N. 17.897 de Humanización de Cárceles, tomando como marco referencial los supuestos teóricos del populismo punitivo y como marco de análisis el modelo de corrientes múltiples de John Kingdon (1995).

Si bien el estado crítico del sistema penitenciario no es algo que se haya logrado superar, para el año 2005, cuando el Frente Amplio gana por primera vez las elecciones nacionales, la inflación penitenciaria venía en ascenso continuo desde el ańo 1995 con la aprobación de la Ley N. ${ }^{\circ} 16.707$ de Seguridad Ciudadana: con capacidad de 3676 plazas, ese ańo llega a albergar a 7213 personas, con las consecuentes problemáticas asociadas a este desborde, junto con la sistemática violación de los derechos básicos y vulneración de las personas. La prisión implica ciertas restricciones, pero corresponde al Estado la tutela y velar por los derechos humanos de la PPL, así como de los funcionarios del sistema. Asimismo, no se habían desarrollado políticas integrales de largo plazo para enfrentar la problemática penitenciaria en su globalidad: las políticas de prevención de delitos a través del endurecimiento de penas, la tipificación de nuevos delitos y el fortalecimiento institucional del Ministerio del Interior eran las estrategias utilizadas. El debate que instala el Frente Amplio con su llegada al gobierno resignifica en buena medida estos procedimientos, además de poner la crisis del sistema en un lugar de atención central y urgente.

Se considera que el punto crítico donde las corrientes se encuentran es la declaración de emergencia humanitaria del sistema por parte del Dr. Tabaré Vázquez en su acto de asunción. Este momento no solamente implica la apertura de la ventana de oportunidad que señala el modelo, sino también da cuenta del cambio de enfoque de las políticas sobre el sistema: se inicia, al menos simbólicamente, un cambio de paradigma sobre la política criminal y también la cárcel. Ya no solo importa la pena asociada al delito cometido, sino también el trato humano y digno a las personas, tanto las privadas de libertad como los funcionarios, y esto, en ese momento, fue una novedad. El Poder Ejecutivo señalaba con absoluta claridad no solamente su preocupación sobre el tema-que bien podría considerarse natural, al ser de izquierda el partido que había asumido el gobierno-, sino también un importantísimo cambio de enfoque de las políticas públicas.

Asimismo, la ley y todo su proceso resultó ser un intento más simbólico que efectivo de seguir un camino diferente a la lógica de la punitividad. La medida estaba orientada a mejorar las condiciones de reclusión y jerarquizar algunas instituciones, y así seguir avanzando en el cambio de la concepción de la política criminal en Uruguay; en eso es de enorme importancia que se haya aprobado. Si bien finalmente sus resultados fueron relativamente magros, efectivamente se liberaron 827 personas que no tuvieron altos índices de reincidencia: $18 \%$, mientras que la media es 60\% (Scapusio, 2008).

Los demás ejes de la ley no llegaron a cumplirse sustancialmente si bien se lograron implementar algunos proyectos laborales gestionados por PPL $\mathrm{e}$ incluso algunos convenios con intendencias departamentales y organismos públicos, además de aumentar la presencia de docentes en los establecimientos penitenciarios. Esto último no es menor en un sistema casi exclusivamente basado en penas privativas de libertad ante cualquier tipo de delito. El hecho de proponer un modelo educativo de cárcel implica un cambio de con- 
cepción fundamental, que pone a la persona como eje del proceso.

También es válido establecer que intervenir solamente en el sistema penitenciario -pues se trata de un subsistema dentro del sistema penal, pese al propósito definido inicialmente de primer paso en un cambio de paradigma- no logró un efecto sobre los problemas que causan la prisionización, mucho menos sobre la reinserción de los liberados. Las cárceles volvieron rápidamente a la situación de origen, por lo que no se trató, a priori, de un proceso políticamente exitoso. Si bien podría considerarse que la aprobación de esta ley supone un avance enorme en materia de derechos humanos, especialmente en incorporar la mirada humanista en los temas relacionados al castigo y de algún modo comprometer a los actores políticos (fundamentalmente al Ministerio del Interior) a mantener esa perspectiva presente en reformas posteriores, los resultados quizás no reflejan plenamente la importancia de este debate, ya que el eje de derechos humanos pasa a ser determinante en la conceptualización de todo el problema de seguridad, no solamente la privación de libertad.

Otra cuestión se plantea: ¿era el momento adecuado para proponer una ley de este tipo? La respuesta es ambigua. Por una parte, es del tipo de medidas que el Frente Amplio podía tomar en un primer período de gobierno, por el clima de adhesión y expectativa que había, y también porque era necesario dar señales de renovación y de cambios de enfoques en algunos paradigmas de políticas públicas. Que finalmente se haya estigmatizado la gestión de seguridad a partir de esta ley, que pone foco en los derechos humanos (pues el discurso que se instala es que había preferencia por los presos y no por las víctimas), indica que faltó acompañarla con un plan de reformas integrales, coordinar con otros actores gubernamentales o de la sociedad civil, a modo de brindar mayor respaldo a la nueva conducción política, así como también una estrategia comunicacional que posibilitara ciertos niveles de consenso sobre la urgencia de humanizar las condiciones de privación de libertad. Ello por cuanto el trato humano a la PPL no se agota dentro del sistema penitenciario ni es solamente responsabilidad de sus funcionarios, y la Ley de Humanización de Cárceles puso esta cuestión en el centro del debate.

\section{Bibliografía}

Bardazano, G.; Corti, A.; Duffau, N. y Trajtemberg, N. (2015). Discutir la cárcel, pensar la sociedad. Contra el sentido común punitivo. Montevideo CSIC, Trilce.

Bentancur, N.; Busquets, J. M. (2016). El decenio progresista. Las políticas públicas de Vázquez a Mujica. Montevideo, Fin de Siglo.

Caetano, G. y Rilla, J. (2004). Historia contemporánea del Uruguay: de la colonia al siglo XXI. Montevideo, Fin de Siglo.

Calderón, R. (2015). Delincuencia, politicas de Estado $y$ derechos humanos. A propósito de la cuestión penitenciaria en Chile. Santiago, Ril Editores.

Díaz, A. (2014). Menores infractores, un problema no menor. Monografía final de grado en Ciencia Política, Montevideo, FCS.

Fassin, D. (2018). Castigar. Buenos Aires, Adriana Hidalgo Editora.

Foucault, M. (2008). Vigilar y castigar. Nacimiento de la prisión. Buenos Aires, Siglo Veintiuno.

Garland, D. (2012). La cultura del control. Crimen y orden social en la sociedad contemporánea. Barcelona, Gedisa.

González, V.; Rijido, E.; Trajtemberg, N. (2015). Sistema penitenciario de Uruguay (1985-2014): cambios, continuidades y desafios. En: Bardazano et al. Discutir la cárcel, pensar la sociedad. Contra el sentido común punitivo. Montevideo, CSIC - Trilce.

Kingdon, J. (2003). Agendas, alternatives, and public policies. New York, Longman.

Pratt, J. (2000). El castigo emotivo y ostentoso. Su declinación y resurgimiento en la sociedad moderna en Punishment and Society, Vol. 2.

Sanjurjo, D. (2019). Gun control policies in Latin America, Palgrave Macmillan. 
Scapusio, B. (2008). El sistema penal uruguayo y su repercusión penitenciaria: la necesidad de su reforma en Ronzoni (comp.). Reforma al sistema penal y carcelario en Uruguay, Montevideo, Fundación Cadal.

Vila, A. (2016). La politica de seguridad ciudadana del mujiquismo: entre acuerdos y disensos. En: Bentancur, et al. El decenio progresista. Las politicas públicas de Vázquez a Mujica. Montevideo, Fin de Siglo.

Walmsley, R. (2018). World Prison Population List (12th edition). Institute for Criminal Policy Research, London.

Zaffaoni, E. R. (2015). La filosofía del sistema penitenciario en el mundo contemporáneo en Bardazano et al.; Discutir la cárcel, pensar la sociedad. Contra el sentido común punitivo. Montevideo, CSIC - Trilce.

Zahariadis, N. (2010). El marco de las corrientes múltiples. Estructuras, limitaciones, perspectivas. En: Sabatier P. A. (ed.). Teoría del proceso de las políticas públicas. Buenos Aires, Argentina: Proyecto de Modernización del Estado, Jefatura de Gabinete de Ministros, Presidencia de la Nación, Argentina. pp. 67-94.

\section{Fuentes documentales}

Informe de la Comisión Honoraria de Mejoramiento del Sistema Carcelario. Artículo 34 de la Ley 16.707 de 1996.

IV Congreso Extraordinario Héctor Rodríguez, 20 y 21 de diciembre de 2003. Disponible en https:// www.frenteamplio.uy/documentos-institucionales/item/131-2003-iv-congreso-extraordinariohector-rodriguez-realizado-los-dias-20-y-21-dediciembre Acceso 21/2/2019.

Discurso del presidente de la República, Tabaré Vázquez, en el acto del Palacio Legislativo. $1 .^{\circ}$ de marzo de 2005. Disponible en http://archivo.presidencia.gub.uy Acceso 4/3/2019.

Proyecto de ley de Libertad Provisional Anticipada: establecimiento de un régimen excepcional. Carpeta 275 , repartido n. 314 de julio 2005. Disponible en https://parlamento.gub.uy/documentosyleyes/ficha-asunto/27103 Acceso 19/4/2019.

Humanización y modernización del sistema carcelario. Carpeta 275, repartido n. ${ }^{\circ} 314$ de julio 2005. Disponible en https://parlamento.gub.uy/ documentosyleyes/ficha-asunto/27103 Acceso 20/4/2019. 